TAIWANESE JOURNAL OF MATHEMATICS

Vol. 11, No. 4, pp. 975-988, September 2007

This paper is available online at http://www.math.nthu.edu.tw/tjm/

\title{
MULTIPLE SOLUTIONS FOR A SECOND-ORDER STURM-LIOUVILLE BOUNDARY VALUE PROBLEM
}

\author{
Yu Tian and Weigao Ge
}

\begin{abstract}
In this paper, we investigate a second-order Sturm-Liouville boundary value problem depending on the parameter $\lambda$. For any $\lambda$ in some explicitly determined open interval, the boundary value problem admits multiple generalized solutions by using very recent three critical points theorems stated in a paper by G. Bonanno (J. Math. Anal. Appl. 29 (2004), 600-614).
\end{abstract}

\section{INTRODUCTION}

In recent years, multiplicity results for nonlinear boundary value problems were extensively studied because of their application in many fields, see [1, 6, 7, 13, 15] and the references therein. The results have been obtained mainly by the fixed point theorem in cones, such as Guo-Krasnosel'skii fixed point theorem in a cone [12], Leggett-Williams fixed point theorem [17], Avery and Henderson's theorem [6]. In the last few years, a result of Ricceri (Theorem 1 of [18]) has been widely used to obtain multiplicity results, see [8-10]. In [19], Ricceri's variational principle was established, that is to say, infinitely many local minima of the functional $\Phi+\lambda \Psi$ for each sufficiently large $\lambda \in R$ exist under some assumptions. Based on the use of Ricceri's variational principle, the exact interval $] \frac{1}{\varphi_{2}(r)}, \frac{1}{\varphi_{1}(r)}[$ is determined in [3] such that for every $\lambda \in] \frac{1}{\varphi_{2}(r)}, \frac{1}{\varphi_{1}(r)}[$, the functional $\Phi+\lambda \Psi$ has at least three critical points. Very recently, in [11] G. Bonanno obtained a new critical point theorem where the coercity and the Palais-Smale condition are not assumed, so further improve the critical point theorem.

We first state the critical point theorem in [11].

Received June 1, 2005, accepted November 18, 2005.

Communicated by Sze-Bi Hsu.

2000 Mathematics Subject Classification: 34C25; 34B15.

Key words and phrases: Critical points, Second-order Sturm-Liouville boundary value problem, $p$ Laplacian.

Supported by National Natural Sciences Foundation of P. R. China (10371006). 
At first, given a real Banach space $X$ and two functions $\Phi, \Psi: X \rightarrow R$, we define the following functionals

$$
\begin{aligned}
& \varphi_{1}(r)=\inf _{x \in \Psi^{-1}(]-\infty, r[)} \frac{\Phi(x)-\inf _{x \in \Psi^{-1}(]-\infty, r[} \omega}{r-\Psi(x)} \Phi(x) \\
& \varphi_{2}\left(r_{1}, r_{2}\right)=\inf _{x \in \Psi^{-1}(]-\infty, r_{1}[)} \sup _{y \in \Psi^{-1}\left(\left[r_{1}, r_{2}[)\right.\right.} \frac{\Phi(x)-\Phi(y)}{\Psi(y)-\Psi(x)}
\end{aligned}
$$

for all $r, r_{1}, r_{2}>\inf _{X} \Psi$, with $r_{1}<r_{2}$, and where ${\overline{\Psi^{-1}(]-\infty, r[)}}^{\omega}$ is the closure of $\Psi^{-1}(]-\infty, r[)$ in the weak topology.

Theorem 1.1. [11] Let $X$ be a reflexive real Banach space, and $\Phi, \Psi$ : $X \rightarrow R$ two sequentially weakly lower semicontinuous and Gâteaux differentiable functionals. Assume that $\Psi$ is (strongly) continuous and satisfies $\lim _{\|x\| \rightarrow+\infty} \Psi(x)=$ $+\infty$. Assume also that there exist two constants $r_{1}$ and $r_{2}$ such that

(j) $\inf _{X} \Psi<r_{1}<r_{2}$;

$(j j) \varphi_{1}\left(r_{1}\right)<\varphi_{2}\left(r_{1}, r_{2}\right)$;

$(j j j) \varphi_{1}\left(r_{2}\right)<\varphi_{2}\left(r_{1}, r_{2}\right)$.

Then, there exists a positive real number $\sigma$ such that, for each

$$
\lambda \in] \frac{1}{\varphi_{2}\left(r_{1}, r_{2}\right)}, \min \left\{\frac{1}{\varphi_{1}\left(r_{1}\right)}, \frac{1}{\varphi_{1}\left(r_{2}\right)}\right\}[,
$$

the equation $\Psi^{\prime}+\lambda \Phi^{\prime}=0$ admits at least two solutions whose norms are less than $\sigma$.

Define

$$
\varphi_{3}\left(r_{1}, r_{2}, r_{3}\right)=\inf _{x \in \Psi^{-1}\left(\left[r_{1}, r_{2}[)\right.\right.} \sup _{y \in \Psi^{-1}\left(\left[r_{2}, r_{3}[)\right.\right.} \frac{\Phi(x)-\Phi(y)}{\Psi(y)-\Psi(x)}
$$

for all $r_{1}, r_{2}, r_{3}>\inf _{X} \Psi$. Clearly, $\varphi_{2}\left(r_{2}, r_{3}\right) \leq \varphi_{3}\left(r_{1}, r_{2}, r_{3}\right)$.

Theorem 1.2. [11] Let $X$ be a reflexive real Banach space, and $\Phi, \Psi: X \rightarrow R$ two sequentially weakly lower semicontinuous and Gâteaux differentiable functionals. Assume that $\Psi$ is (strongly) continuous and satisfies $\lim _{\|x\| \rightarrow+\infty} \Psi(x)=+\infty$. Assume also that there exists three constants $r_{1}, r_{2}$ and $r_{3}$ such that

$(k) \inf _{X} \Psi<r_{1}<r_{2}<r_{3}$;

$(k k) \max \left\{\varphi_{1}\left(r_{1}\right), \varphi_{1}\left(r_{2}\right), \varphi_{1}\left(r_{3}\right)\right\}<\min \left\{\varphi_{2}\left(r_{1}, r_{2}\right), \varphi_{3}\left(r_{1}, r_{2}, r_{3}\right)\right\}$. 
Then there exists a positive real number $\sigma$ such that for each

$$
\lambda \in] \max \left\{\frac{1}{\varphi_{2}\left(r_{1}, r_{2}\right)}, \frac{1}{\varphi_{3}\left(r_{1}, r_{2}, r_{3}\right)}\right\}, \min \left\{\frac{1}{\varphi_{1}\left(r_{1}\right)}, \frac{1}{\varphi_{1}\left(r_{2}\right)}, \frac{1}{\varphi_{1}\left(r_{3}\right)}\right\}[,
$$

the equation $\Psi^{\prime}+\lambda \Phi^{\prime}=0$ admits at least three solutions whose norms are less than $\sigma$.

The existence of multiple solutions for Dirichlet boundary value problem were studied in [11] by means of Theorem 1.1 and Theorem 1.2. This paper is a continuation for the application of the critical point theorem.

We consider the existence of solutions for the following second-order SturmLiouville boundary value problem:

$$
\left\{\begin{array}{l}
\left(\Phi_{p}\left(x^{\prime}\right)\right)^{\prime}+\lambda f(t, x) g\left(x^{\prime}\right)=0, \quad t \in[a, b], \\
\alpha x(a)-\beta x^{\prime}(a)=0, \quad \gamma x(b)+\eta x^{\prime}(b)=0,
\end{array}\right.
$$

where $\alpha, \gamma \geq 0, \beta, \eta>0, \Phi_{p}(x):=|x|^{p-2} x, p>1, f$ is an $L^{1}$-Carathedory function, $g: R \rightarrow R$ is a bounded continuous function such that $0<m:=$ $\inf g, \quad 0<M:=\sup g$.

For $\beta=0, \eta=0$ (1.4) becomes Dirichlet boundary value problem, which has been studied extensively in recent years, see $[4,11,14]$ and the references therein. For $p=2, \beta=0, \eta=0,(1.4)$ has been studied in [1,22]. For $\beta=0, \gamma=0,(1.4)$ becomes mixed boundary value problem, which was studied in [5] by using critical point theorem [3]. By using cone expansion-compression fixed-point theorem, Erbe and Wang studied in [16] the equation $u^{\prime \prime}+g(t) f(u)=0$ and Sturm-Liouville boundary condition, and get existence result by imposing the following conditions on $f$ :

(a) $\lim _{x \rightarrow 0+} \frac{f(x)}{x}=0$ and $\lim _{x \rightarrow \infty} \frac{f(x)}{x}=+\infty$, (superliner);

or

(b) $\lim _{x \rightarrow 0+} \frac{f(x)}{x}=+\infty$ and $\lim _{x \rightarrow \infty} \frac{f(x)}{x}=0$, (suberliner).

In this paper, we consider the existence of solutions for problem (1.4). Due to $\beta, \eta \neq 0$ in boundary condition, the Banach space $X$ and functionals $\Phi, \Psi$ defined on $X$ are different from [11]. Our approach is based on Theorem 1.1 and 1.2 and enables us to obtain multiple solutions of (1.4). Our results extend and complement many results in the literature $[2,5,11,16]$.

This paper is organized as follows. in section 2 , we introduce some basic definition and notation. In section 3, the variational approach is justified and the regularity of an appropriate functional involved is proved. In section 4, existence results are given. 


\section{Basic Definitions and Notations}

The Sobolev space $W^{1, p}([a, b])=\left\{u: u \in C([a, b]), u^{\prime} \in L^{p}([a, b])\right\}, 1<p<$ $\infty$. The norm over $W^{1, p}([a, b])$ is defined by $\|u\|_{W^{1, p}}=\left(\int_{a}^{b}|u(t)|^{p}+\left|u^{\prime}(t)\right|^{p} d t\right)^{\frac{1}{p}}$. It is well known that $W^{1, p}([a, b])$ is a reflexive Banach space.

In this paper, we need the following notations: $\|u\|_{\infty}=\max _{t \in[a, b]}|u(t)|,\|u\|_{L^{p}}=$ $\left(\int_{a}^{b}|u(t)|^{p} d t\right)^{\frac{1}{p}}$

Definition 2.1. A function $f:[a, b] \times R \rightarrow R$ is said to be $L^{1}$-Carathedory if

(a) $t \rightarrow f(t, u)$ is measurable for every $u \in R$;

(b) $u \rightarrow f(t, u)$ is continuous for almost every $t \in[a, b]$;

(c) for every $\rho>0$ there exists a function $l_{\rho} \in L^{1}([a, b])$ such that

$$
\sup _{|u|<\rho}|f(t, u)| \leq l_{\rho}(t) \text { for almost every } t \in[a, b] .
$$

Definition 2.2. A function $u:[a, b] \rightarrow R$ is said a generalized solution to (1.4) if $u \in C^{1}([a, b]), \Phi_{p}\left(u^{\prime}\right) \in A C([a, b])$ satisfies Sturm-Liouville boundary condition and

$$
\left(\Phi_{p}\left(u^{\prime}\right)\right)^{\prime}+\lambda f(t, u) g\left(u^{\prime}\right)=0
$$

for almost every $t \in[a, b]$.

For $p>1$, define $J: R \rightarrow R$ by putting, for any $x \in R$

$$
J(x):=\int_{0}^{x} \frac{(p-1)|s|^{p-2}}{g(s)} d s,
$$

clearly $J$ is an increasing homeomorphism on $R$ and by [4] one has

$$
\frac{|x|^{p-2} x}{M} \leq J(x) \leq \frac{|x|^{p-2} x}{m} \text { for all } x \in R, \quad \frac{d}{d t} J(x(t))=\frac{\frac{d}{d t} \Phi_{p}(x(t))}{g(x(t))} .
$$

Definition 2.3. A function $u:[a, b] \rightarrow R$ is said a weak solution to (1.4) if $u \in W^{1, p}([a, b])$ and

$$
\begin{aligned}
& \int_{a}^{b} J\left(u^{\prime}(t)\right) v^{\prime}(t) d t+J\left(\frac{\alpha u(a)}{\beta}\right) v(a)-J\left(-\frac{\gamma u(b)}{\eta}\right) v(b) \\
& =\int_{a}^{b} \lambda f(t, u(t)) v(t) d t
\end{aligned}
$$


for every $v \in W^{1, p}([a, b])$.

We claim that generalized solutions to problem (1.4) coincide with weak ones when $f$ is an $L^{1}$-Carathedory function. In fact, if $u \in C^{1}([a, b])$ is a generalized solution, it is clear $u \in W^{1, p}([a, b])$. Since $g: R \rightarrow R$ is a continuous function and inf $g>0$ we have

$$
\frac{\left(\Phi_{p}\left(u^{\prime}(t)\right)\right)^{\prime}}{g\left(u^{\prime}(t)\right)}+\lambda f(t, u(t))=0
$$

Multiplying (2.5) by $v \in W^{1, p}([a, b])$, then integrating them on $[a, b]$, in view of the boundary condition, we obtain (2.4). On the other hand, if $u$ is a weak solution, by integrating by parts and (2.3) we have

$$
\begin{aligned}
0= & \int_{a}^{b} J\left(u^{\prime}(t)\right) v^{\prime}(t) d t+J\left(\frac{\alpha x u(a)}{\beta}\right) v(a) \\
& -J\left(-\frac{\gamma u(b)}{\beta}\right) v(b)-\int_{a}^{b} \lambda f(t, u(t)) v(t) d t \\
= & J\left(u^{\prime}(b)\right) v(b)-J\left(u^{\prime}(a)\right) v(a)-\int_{a}^{b} \frac{\left(\Phi_{p}\left(u^{\prime}(t)\right)\right)^{\prime}}{g\left(u^{\prime}(t)\right)} v(t) d t \\
& +J\left(\frac{\alpha x u(a)}{\beta}\right) v(a)-J\left(-\frac{\gamma u(b)}{\eta}\right) v(b)-\int_{a}^{b} \lambda f(t, u(t)) v(t) d t .
\end{aligned}
$$

i.e.

$$
\begin{aligned}
0 & =\left[J\left(u^{\prime}(b)\right)-J\left(-\frac{\gamma u(b)}{\eta}\right)\right] v(b)+\left[-J\left(u^{\prime}(a)\right)+J\left(\frac{\alpha u(b)}{\beta}\right)\right] v(a) \\
& -\int_{a}^{b}\left\{\frac{\left(\Phi_{p}\left(u^{\prime}(t)\right)\right)^{\prime}}{g\left(u^{\prime}(t)\right)}+\lambda f(t, u(t))\right\} v(t) d t
\end{aligned}
$$

holds for any $v \in W^{1, p}([a, b])$. and hence for all $v \in C^{\infty}([a, b])$. Thus, by fundamental lemma of variational, $x$ satisfies the equation (2.5) for a.e. $t \in[a, b]$. Then (2.6) become

$$
\left[J\left(u^{\prime}(b)-J\left(-\frac{\gamma u(b)}{\eta}\right)\right]=v(b)+\left[-J\left(u^{\prime}(a)\right)+J\left(\frac{\alpha u(b)}{\beta}\right)\right] v(a)=0\right.
$$

for all $v \in W^{1, p}([a, b])$. We will show $x$ satisfies boundary condition in BVP (1.4). If not, without loss of generality, we assume

$$
\gamma u(b)+\eta u^{\prime}(b)>0,
$$

which means $J\left(u^{\prime}(b)\right)-J\left(-\frac{\gamma u(b)}{\eta}\right)>0$. Let $v(t)=t-a \in C^{\infty}([a, b]) \subset$ $W^{1, p}([a, b])$, then $\left[J\left(u^{\prime}(b)\right)-J\left(-\frac{\gamma u(b)}{\eta}\right)\right](b-a)>0$, a contralicaion. Therefore, $u$ is a generalized solution of problem (1.4). 


\section{Preliminaries}

For each $u \in W^{1, p}([a, b])$, put

$$
\Psi(u)=\int_{a}^{b}\left(\int_{0}^{u^{\prime}(t)} J(s) d s\right) d t+\frac{\beta}{\alpha} \int_{0}^{\frac{\alpha u(a)}{\beta}} J(s) d s+\frac{\eta}{\gamma} \int_{0}^{-\frac{\gamma u(b)}{\eta}} J(s) d s
$$

and

$$
\Phi(u):=-\int_{a}^{b} F(t, u(t)) d t
$$

where $F(t, \xi)=\int_{0}^{\xi} f(t, x) d x$ for $t \in[a, b]$. By (2.3), via a simple calculation, we have

$$
\begin{aligned}
& \frac{1}{M p}\left[\left\|u^{\prime}\right\|_{L^{p}}^{p}+\frac{\alpha^{p-1}}{\beta^{p-1}}|u(a)|^{p}+\frac{\gamma^{p-1}}{\eta^{p-1}}|u(b)|^{p}\right] \\
& \leq \Psi(u) \leq \frac{1}{m p}\left[\left\|u^{\prime}\right\|_{L^{p}}^{p}+\frac{\alpha^{p-1}}{\beta^{p-1}}|u(a)|^{p}+\frac{\gamma^{p-1}}{\eta^{p-1}}|u(b)|^{p}\right] .
\end{aligned}
$$

Lemma 3.1. If $u \in W^{1, p}([a, b])$, and there exists $r>0$ such that $\Psi(u) \leq r$, then

$$
\|u\|_{\infty} \leq \sqrt[p]{M p r}\left(\sqrt[p]{\frac{\beta^{p-1}}{\alpha^{p-1}}}+(b-a)^{\frac{1}{q}}\right)
$$

Proof. If $u \in W^{1, p}([a, b])$, it follows from the mean value theorem that

$$
|u(t)|=\left|u(a)+\int_{a}^{t} u^{\prime}(s) d s\right| \leq|u(a)|+\left\|u^{\prime}\right\|_{L^{p}}(b-a)^{\frac{1}{q}} .
$$

In view of $\Psi(u) \leq r$ and (3.3) we have

$$
\frac{1}{M p}\left[\left\|u^{\prime}\right\|_{L^{p}}^{p}+\frac{\alpha^{p-1}}{\beta^{p-1}}|u(a)|^{p}+\frac{\gamma^{p-1}}{\eta^{p-1}}|u(b)|^{p}\right] \leq r,
$$

then

$$
\left\|u^{\prime}\right\|_{L^{p}} \leq \sqrt[p]{M p r}, \quad|u(a)| \leq \sqrt[p]{\frac{\beta^{p-1}}{\alpha^{p-1}} M p r}
$$

The conclusion then follows from (3.5). 
Lemma 3.2. Let $\Psi: W^{1, p}([a, b]) \rightarrow R$ be defined as (3.1). Then $\Psi$ : $W^{1, p}([a, b]) \rightarrow R$ is a sequentially weakly lower semicontinuous and continuous functional and $\lim _{\|u\| \rightarrow+\infty} \Psi(u)=+\infty$, whose Gâteaux derivative at the point $u$ is the functional $\Psi^{\prime}(u) \in\left(W^{1, p}([a, b])\right)^{*}$ given by

$$
\Psi^{\prime}(u)(v)=\int_{a}^{b} J\left(u^{\prime}(t)\right) v^{\prime}(t) d t+J\left(\frac{\alpha u(a)}{\beta}\right) v(a)-J\left(-\frac{\gamma u(b)}{\eta}\right) v(b)
$$

for every $v \in W^{1, p}([a, b])$.

Proof. First we will show that $\Psi$ is a continuous functional. Let $u_{n} \rightarrow u$ on $W^{1, p}([a, b])$ as $n \rightarrow \infty$, then $\left\|u_{n}^{\prime}-u^{\prime}\right\|_{L^{p}} \rightarrow 0$ and $u_{n}(a) \rightarrow u(a), u_{n}(b) \rightarrow u(b)$ as $n \rightarrow \infty$.

$$
\begin{aligned}
& \left|\Psi\left(u_{n}\right)-\Psi(u)\right| \leq \int_{a}^{b}\left|\int_{u^{\prime}(t)}^{u_{n}^{\prime}(t)} J(s) d s\right| d t+\frac{\beta}{\alpha}\left|\int_{\frac{\alpha u_{n}(a)}{\beta}}^{\frac{\alpha u_{n}(a)}{\beta}} J(s) d s\right| \\
& +\frac{\eta}{\gamma}\left|\int_{-\frac{\gamma u_{n}(b)}{\eta}}^{-\frac{\gamma u_{n}(b)}{\eta}} J(s) d s\right|
\end{aligned}
$$

by (2.3), we have

$$
\begin{aligned}
\int_{a}^{b}\left|\int_{u^{\prime}(t)}^{u_{n}^{\prime}(t)} J(s) d s\right| d t & \leq\left.\frac{1}{m} \int_{a}^{b}\left|\int_{u^{\prime}(t)}^{u_{n}^{\prime}(t)}\right| s\right|^{p-2} s d s \mid d t \\
& =\left.\frac{1}{m p} \int_{a}^{b}|| u^{\prime}(t) u_{n}^{\prime}(t)\right|^{p}-\left|u^{\prime}(t)^{p}\right| \mid d t
\end{aligned}
$$

Let $\psi(x)=|x|^{p}$, by mean value theorem at $u_{n}^{\prime}(t)$ and exists $0<\xi(t)<1$ such that

$$
\left|\psi\left(u_{n}^{\prime}(t)\right)-\psi\left(u^{\prime}(t)\right)\right|=\left|\psi^{\prime}\left(u_{n}^{\prime}(t)\right)+\xi(t)\left(u_{n}^{\prime}(t)\right)-\left(u^{\prime}(t)\right) \times\left(u_{n}^{\prime}(t)\right)-\left(u^{\prime}(t)\right)\right|,
$$

i.e.

$$
\begin{aligned}
||\left(\left.u_{n}^{\prime}(t)\right|^{p}-\mid\left(\left.u^{\prime}(t)\right|^{p} \mid\right.\right. & =p \mid \Phi_{p}\left(u_{n}^{\prime}(t)\right)+\xi(t)\left(u_{n}^{\prime}(t)-\left(u^{\prime}(t)\right)|\times| u_{n}^{\prime}(t)-u^{\prime}(t) \mid\right. \\
& \leq p\left(\left|u_{n}^{\prime}(t)\right|+\left|u_{n}^{\prime}(t)-\left(u^{\prime}(t) \mid\right)^{p-1}\right| u_{n}^{\prime}(t)-u^{\prime}(t) \mid\right. \\
& \leq k\left[\left|u_{n}^{\prime}(t)\right|^{p-1}\left|u_{n}^{\prime}(t)-u^{\prime}(t)\right|+\left|u_{n}^{\prime}(t)-u^{\prime}(t)\right|^{p}\right]
\end{aligned}
$$

Therefore

$$
\begin{aligned}
& \int_{a}^{b}\left|\int_{u^{\prime}(t)}^{u_{n}^{\prime}(t)} J(s) d s\right| d t \\
\leq & \frac{k}{m p}\left(\int_{a}^{b}\left|u^{\prime}(t)\right|^{p-1}\left|u_{n}^{\prime}(t)-u^{\prime}(t)\right|+\left|u_{n}^{\prime}(t)-u^{\prime}(t)\right|^{p} d t\right) \\
\leq & \frac{k}{m p}\left(\left\|u^{\prime}(t)\right\|_{L p}^{\frac{p}{q}}\left\|u^{\prime}(t)-u^{\prime}\right\|_{L P}+\left\|u^{\prime}(t)-u^{\prime}\right\|_{L P}^{p}\right) \\
& \rightarrow 0 \text { as } u_{n} \rightarrow u \text { on } W^{1, p}([a, b]) .
\end{aligned}
$$


Moreover, by (2.3)

$$
\begin{aligned}
& \left|\int_{\frac{\alpha u(a)}{\beta}}^{\frac{\alpha u_{n}(a)}{\beta}} J(s) d s\right| \\
\leq & \left.\frac{1}{m}\left|\int_{\frac{\alpha u(a)}{\beta}}^{\frac{\alpha u_{n}(a)}{\beta}}\right| s\right|^{p-2} s d s\left|\leq \frac{1}{m p}\right|\left|\frac{\alpha u_{n}(a)}{\beta}\right|^{p}-\left|\frac{\alpha u(a)}{\beta}\right|^{p} \mid \\
= & \left.\frac{\alpha^{p}}{\beta^{p} m p}|| u_{n}(a)\right|^{p}-|u(a)|^{p} \mid \rightarrow 0 \text { as } u_{n} \rightarrow u \text { on } W^{1, p}([a, b])
\end{aligned}
$$

and

$$
\left|\int_{-\frac{\gamma u(b)}{\eta}}^{-\frac{\gamma u_{n}(a)}{\eta}} J(s) d s\right| \rightarrow 0 \text { as } u_{n} \rightarrow u
$$

It follows from (3.6) (3.8)-(3.10)that $\Psi\left(u_{n}\right) \rightarrow \Psi(u)$ as $u_{n} \rightarrow u$. Thus we have shown that $\Psi$ is continuous, taking into account that $\Psi$ is convex, from (Proposition 25.20 [20]) we obtain that $\Psi$ is a sequentially weakly lower semi-continuous functional.

Now let $\|u\| \rightarrow \infty$, and consider two cases: (i) $\left\|u^{\prime}\right\|_{L^{p}} \rightarrow \infty$, (ii) $\|u\|_{L^{p}} \rightarrow \infty$.

(i) By (3.3) we have $\Psi(u) \rightarrow \infty$.

(ii) In view of $\|u\|_{L^{p}} \leq(b-a)^{\frac{1}{p}}\|u\|_{\infty}$ we have $\|u\|_{\infty} \rightarrow \infty$, together with continuity of $u$ on $[a, b]$ we get $u(a) \rightarrow \infty$ or $u(b) \rightarrow \infty$, therefore $\Psi(u) \rightarrow$ $\infty$. So $\lim _{\|u\| \rightarrow \infty} \Psi(u)=\infty$.

By the definition of Fréchet derivative, it is easy to see that

$$
\Psi^{\prime}(u)(v)=\int_{a}^{b} J\left(u^{\prime}(t)\right) v^{\prime}(t) d t+J\left(\frac{\alpha u(a)}{\beta}\right) v(a)-J\left(-\frac{\gamma u(b)}{\eta}\right) v(b) .
$$

for every $v \in W^{1, p}([a, b])$. The proof is complete.

Remark 3.1. If $u \in W^{1, p}([a, b])$ is a critical point of $\Psi+\lambda \Phi$, in view of Definition 2.3, then $u$ is a weak solution of problem (1.4).

\section{MAIN Results}

Theorem 4.1. Let $f:[a, b] \times R \rightarrow R$ be an $L^{1}$-Carathedory function, and put $F(t, \xi)=\int_{0}^{\xi} f(t, x) d x$ for every $(t, \xi) \in[a, b] \times R$. Assume there exist two positive constants $0<c_{1}<c_{2}$ and a function $y^{*} \in W^{1, p}([a, b])$ such that 
(H1) $c_{1}^{p} \leq\left\{\left\|y^{*^{\prime}}\right\|_{L^{p}}^{p}+\frac{\alpha^{p-1}}{\beta^{p-1}}\left|y^{*}(a)\right|^{p}+\frac{\gamma^{p-1}}{\eta^{p-1}}\left|y^{*}(b)\right|^{p}\right\}\left[(b-a)^{\frac{1}{q}}+\sqrt[p]{\frac{\beta^{p-1}}{\alpha^{p-1}}}\right]^{p} \leq \frac{m}{M} c_{2}^{p} ;$

(H2) $A_{c_{i}} M p\left[(b-a)^{\frac{1}{q}}+\sqrt[p]{\frac{\beta^{p-1}}{\alpha^{p-1}}}\right]^{p}<\frac{\int_{a}^{b} F\left(t, y^{*}(t)\right) d t-\int_{a}^{b} \sup _{|\xi| \leq c_{1}} F(t, \xi) d t}{\Psi\left(y^{*}\right)}$ for $i=1,2$.

Then for each

$$
\lambda \in] \frac{\Psi\left(y^{*}\right)}{\int_{a}^{b} F\left(t, y^{*}(t)\right) d t-\int_{a}^{b} \sup _{|\xi| \leq c_{1}} F(t, \xi) d t}, \frac{\min \left\{\frac{1}{A_{c_{1}}}, \frac{1}{A_{c_{2}}}\right\}}{M p\left[(b-a)^{\frac{1}{q}}+\sqrt[p]{\frac{\beta^{p-1}}{\alpha^{p-1}}}\right]^{p}}[,
$$

the problem (1.4) has at least two generalized solutions whose norms in $C([a, b])$ are less than $c_{2}$, where $A_{c}=\frac{1}{c^{p}} \int_{a}^{b} \sup _{|\xi| \leq c} F(t, \xi) d t$.

Proof. Take the Banach space $X=W^{1, p}([a, b])$. The functional $\Phi, \Psi$ have been defined as in (3.1) and (3.2). It is well known $X$ is a reflexive Banach space. Lemma 3.2 and [4] have showed that $\Phi, \Psi: X \rightarrow R$ are two sequentially weakly lower semicontinuous and Gâteaux differentiable functionals and that $\Psi$ is continuous and satisfies $\lim _{\|x\| \rightarrow+\infty} \Psi(x)=+\infty$.

We will obtain at least two critical points of $\Psi+\lambda \Phi$ by applying Theorem 1.1. It remains to verify conditions (j)(jj)(jjj) in Theorem 1.1.

Let $r_{i}=\frac{c_{i}^{p}}{M p}\left((b-a)^{\frac{1}{q}}+\sqrt[p]{\frac{\beta^{p-1}}{\alpha^{p-1}}}\right)^{-p}, i=1,2$. By (3.3) and (H1) we have

$$
\Psi\left(y^{*}\right) \leq \frac{1}{m p}\left[\left\|y^{*^{\prime}}\right\|_{L^{p}}^{p}+\frac{\alpha^{p-1}}{\beta^{p-1}}\left|y^{*}(a)\right|^{p}+\frac{\gamma^{p-1}}{\eta^{p-1}}\left|y^{*}(b)\right|^{p}\right]<r_{2}
$$

and

$$
\Psi\left(y^{*}\right) \geq \frac{1}{M p}\left[\left\|y^{*^{\prime}}\right\|_{L^{p}}^{p}+\frac{\alpha^{p-1}}{\beta^{p-1}}\left|y^{*}(a)\right|^{p}+\frac{\gamma^{p-1}}{\eta^{p-1}}\left|y^{*}(b)\right|^{p}\right]>r_{1} .
$$

It is easy to verify that (j) holds since $r_{1}, r_{2}>0$.

We will now show that (jj) in Theorem 1.1 is satisfied.

Taking into account that the function $u \equiv 0$ on $[a, b]$ obviously belongs to $\Psi^{-1}(]-\infty, r[)$ and that $\Psi(0)=\Phi(0)=0$, we get

$$
\begin{aligned}
\varphi_{1}(r) & =\inf _{x \in \Psi^{-1}(]-\infty, r[)} \frac{\Phi(x)-\inf _{x \in \bar{\Psi}^{-1}(]-\infty, r[)} \omega(x)}{r-\Psi(x)} \\
& \leq-\frac{1}{r}{ }_{x \in \Psi^{-1}(]-\infty, r[)} \omega(x) .
\end{aligned}
$$


\left.\left. Noticing ${\overline{\Psi^{-1}(]-\infty, r[)^{\omega}}}^{\omega}=\Psi^{-1}(]-\infty, r\right]\right)$, by Lemma 3.1 we obtain

$$
\varphi_{1}(r) \leq \frac{1}{r} \int_{a}^{b} \sup _{|\xi| \leq \sqrt[p]{M p r}\left[(b-a)^{\frac{1}{q}}+\sqrt[p]{\frac{\beta^{p-1}}{\alpha^{p-1}}}\right]} F(t, \xi) d t .
$$

So

$$
\varphi_{1}\left(r_{i}\right) \leq \frac{M p\left[(b-a)^{\frac{1}{q}}+\sqrt[p]{\frac{\beta^{p-1}}{\alpha^{p-1}}}\right]^{p}}{c_{i}^{p}} \int_{a}^{b} \sup _{|\xi| \leq c_{i}} F(t, \xi) d t, \quad i=1,2 .
$$

On the other hand, by Lemma 3.1 and $r_{1} \leq \Psi\left(y^{*}\right) \leq r_{2}$ we have

$$
\begin{aligned}
& \varphi_{2}\left(r_{1}, r_{2}\right)=\inf _{x \in \Phi^{-1}(]-\infty, r_{1}[)} \sup _{y \in \Psi^{-1}\left(\left[r_{1}, r_{2}[)\right.\right.} \frac{\Phi(x)-\Phi(y)}{\Psi(y)-\Psi(x)} \\
\geq & \inf _{x \in \Phi^{-1}(]-\infty, r_{1}[)} \frac{\Phi(x)-\Phi\left(y^{*}\right)}{\Psi\left(y^{*}\right)-\Psi(x)} \\
\geq & \inf _{x \in \Phi^{-1}(]-\infty, r_{1}[)} \frac{1}{\Psi\left(y^{*}\right)-\Psi(x)}\left(\int_{a}^{b} F\left(t, y^{*}(t)\right) d t-\int_{a}^{b} F(t, x(t)) d t\right)
\end{aligned}
$$

By (H2) we have that $\int_{a}^{b} F\left(t, y^{*}(t)\right) d t-\int_{a}^{b} F(t, x(t)) d t>0$, so

$$
\varphi_{2}\left(r_{1}, r_{2}\right) \geq \frac{1}{\Psi\left(y^{*}\right)}\left(\int_{a}^{b} F\left(t, y^{*}(t)\right) d t-\int_{a}^{b} \sup _{|\xi| \leq c_{1}} F(t, \xi) d t\right) .
$$

From (H2) we have (jj) (jjj) in Theorem 1.1 hold. By choosing $\sigma=r_{2}$, the conclusion follows.

In Theorem 4.1, condition (H2) is related to the function $y^{*} \in W^{1, p}$. A different function $y^{*} \in W^{1, p}$ would lead to a different condition, which is similar to (H2). For example, we let $y^{*}(t)=d(t-a)$, where $d$ is a constant. We have the following result.

Corollary 4.2. Let $f:[a, b] \times R \rightarrow R$ be an $L^{1}$-Carathedory function, and put $F(t, \xi)=\int_{0}^{\xi} f(t, x) d x$ for every $(t, \xi) \in[a, b] \times R$. Assume there exist three positive constants $c_{1}, d, c_{2}$ such that

$$
\begin{aligned}
& (H 1)^{\prime} \quad c_{1}<d\left[(b-a)+\sqrt[p]{\frac{\gamma^{p-1}}{\eta^{p-1}}}\right]^{p}\left[(b-a)^{\frac{1}{q}}+\sqrt[p]{\frac{\beta^{p-1}}{\alpha^{p-1}}}\right]<\sqrt[p]{\frac{m}{M}} c_{2} \\
& (H 2)^{\prime} \quad A_{c_{i}} \frac{M}{m}\left((b-a)^{\frac{1}{q}}+\sqrt[p]{\frac{\beta^{p-1}}{\alpha^{p-1}}}\right)^{p}\left[b-a+\frac{\gamma^{p-1}}{\eta^{p-1}}(b-a)^{p}\right]<B\left(d(t-a), d, c_{1}\right) \\
& \quad \text { for } i=1,2
\end{aligned}
$$


where $A_{c}$ is defined in Theorem 4.1 and

$$
B\left(d(t-a), d, c_{1}\right)=\frac{1}{d^{p}}\left[\int_{a}^{b} F(t, d(t-a)) d t-\int_{a}^{b} \sup _{|\xi| \leq c_{1}} F(t, \xi) d t\right] .
$$

Then for each

$$
\lambda \in] \frac{b-a+\frac{\gamma^{p-1}}{\eta^{p-1}}(b-a)^{p}}{m p B\left(d(t-a), d, c_{1}\right)}, \frac{\min \left\{\frac{1}{A_{c_{1}}}, \frac{1}{A_{c_{2}}}\right\}}{M p\left[(b-a)^{\frac{p}{q}}+\sqrt[p]{\frac{\beta^{p-1}}{\alpha^{p-1}}}\right]^{p}},
$$

the problem (1.4) has at least two generalized solutions whose norms in $C([a, b])$ are less than $c_{2}$.

Example 4.1. Let $p=2, \alpha=1, \beta=1, \gamma=3, \eta=1, g:[0,1] \rightarrow R$ be defined by $g(x)=1+|\sin x|$ for every $x \in R, \inf g=m=1, \sup g=M=2$. Moreover, let $f: R \rightarrow R$ be the function defined as follows

$$
f(x)=\left\{\begin{array}{l}
1, \quad x \in]-\infty, 1], \\
\left.\left.x^{10}, \quad x \in\right] 1,4\right], \\
\left.\left.4^{10}(-x+5), \quad x \in\right] 4,5\right], \\
0, \quad x \in] 5,40], \\
\left.f^{*}(x), \quad x \in\right] 40,+\infty[,
\end{array}\right.
$$

where $f^{*}$ is an arbitrary function on $] 40,+\infty\left[\right.$ satisfying $f^{*}(40)=0$. It is easy to verify all assumptions of Corollary 4.2 by choosing $c_{1}=1, d=5, c_{2}=40$. Therefore, thanks to Corollary 4.2 for each $\lambda \in] \frac{50}{4^{10}}, \frac{200}{4^{10}}[$, the problem

$$
\left\{\begin{array}{l}
x^{\prime \prime}+\lambda f(x)\left(1+\left|\sin x^{\prime}\right|\right)=0, \\
x(0)-x^{\prime}(0)=0, \quad 3 x(1)+x^{\prime}(1)=0
\end{array}\right.
$$

admits at least two classical solutions whose norms in $C([0,1])$ are less than 40 . We denote the two solutions as $x_{1}, x_{2}$, by $\lambda>0, f\left(x_{i}\right) \geq 0$ and $g(x)>0$, we have $x_{i}^{\prime \prime}(t) \leq 0$ on $t \in[0,1], i=1,2$, which together with $x_{i}(0)-x_{i}^{\prime}(0)=$ $0,3 x_{i}(1)+x_{i}^{\prime}(1)=0$ shows that $x_{i}(t) \geq 0$ on $[0,1]$.

Remark 4.1. If we are applying some cone expansion-compression fixed point theorem [12], then some superlinear or sublinear assumptions on $f$ must be imposed in order to obtain existence results, see [16]. In our approach, we do not need such assumptions, as Example 4.1 shows. 
Theorem 4.3. Assume that there exist five constants $c_{1}, d_{1}, c_{2}, d_{2}, c_{3}$ with $c_{i}<d_{i}\left[(b-a)^{\frac{1}{q}}+\sqrt[p]{\frac{\beta^{p-1}}{\alpha^{p-1}}}\right]^{p}\left[b-a+\frac{\gamma^{p-1}}{\eta^{p-1}}(b-a)^{p}\right]^{\frac{1}{p}} \leq c_{i+1} \sqrt[p]{\frac{m}{M}}, i=1,2$

such that

$$
\begin{aligned}
& \frac{M}{m}\left[\sqrt[p]{\frac{\beta^{p-1}}{\alpha^{p-1}}}+(b-a)^{\frac{1}{q}}\right]^{p}\left[b-a+\frac{\gamma^{p-1}}{\eta^{p-1}}(b-a)^{p}\right] \\
& A^{*}\left(c_{1}, c_{2}, c_{3}\right) \leq B_{c_{1}, c_{2}}^{*}\left(d_{1}, d_{2}\right),
\end{aligned}
$$

where $A^{*}\left(c_{1}, c_{2}, c_{3}\right)=\max \left\{A_{c_{i}}: i=1,2,3\right\}, B_{c_{1}, c_{2}}^{*}\left(d_{1}, d_{2}\right)=\min \left\{B\left(d_{1}(t-\right.\right.$ $\left.\left.a), c_{1}\right), B\left(d_{2}(t-a), c_{2}\right)\right\}$. Then for each

$$
\lambda \in] \frac{b-a+\frac{\gamma^{p-1}}{\eta^{p-1}}(b-a)^{p}}{m p B_{c_{1}, c_{2}}^{*}\left(d_{1}, d_{2}\right)}, \frac{1}{M p A^{*}\left(c_{1}, c_{2}, c_{3}\right)}\left[(b-a)^{\frac{1}{q}}+\sqrt[p]{\frac{\beta^{p-1}}{\alpha^{p-1}}}\right]^{-p}[,
$$

problem (1.4) has at least two nonzero generalized solutions whose norms in $C([a, b])$ are less than $c_{3}$.

Proof. Take the Banach space $X$ and the functionals $\Psi, \Phi$ on $X$ are as in Section 2 and in (3.1)(3.2). Let $r_{i}=\frac{c_{i}^{p}}{M p}\left((b-a)^{\frac{1}{q}}+\sqrt[p]{\frac{\beta^{p-1}}{\alpha^{p-1}}}\right)^{-p}, i=1,2,3$ and $y_{1}(t)=d_{1}(t-a), y_{2}(t)=d_{2}(t-a)$. Arguing as in the proof of Theorem 4.1, one has

$$
\begin{aligned}
r_{1} & <\Psi\left(y_{1}\right)<r_{2}<\Psi\left(y_{2}\right)<r_{3}, \\
\varphi_{2}\left(r_{1}, r_{2}\right) & \geq \frac{m p}{d_{1}^{p}+\frac{\gamma^{p-1}}{\eta^{p-1}} d_{1}^{p}(b-a)^{p}} B\left(d_{1}(t-a), d_{1}, c_{1}\right), \\
\varphi_{3}\left(r_{1}, r_{2}, r_{3}\right) & \geq \varphi\left(r_{2}, r_{3}\right) \geq \frac{m p}{d_{1}^{p}+\frac{\gamma^{p-1}}{\eta^{p-1}} d_{1}^{p}(b-a)^{p}} B\left(d_{2}(t-a), d_{2}, c_{2}\right), \\
\varphi\left(r_{i}\right) & \leq M p\left[(b-a)^{\frac{1}{q}}+\sqrt[p]{\frac{\beta^{p-1}}{\alpha^{p-1}}}\right]^{p} A_{c_{i}}, i=1,2,3 .
\end{aligned}
$$

Therefore, taking into account (4.3), from Theorem 1.2, there exist at least three generalized solutions. Not taking into account the zero solution, there are at least two nonzero generalized solutions whose norms in $C([a, b])$ are less than $c_{3}$. 


\section{ACKNOWLEDGMENT}

The authors thank the referees for their valuable comments and suggestions on the manuscript.

\section{REFERENCES}

1. R. P. Agarwal, D. O'Regan and P. J. Y. Wong, Positive Solutions of Differential, Difference and Integral Equations, Kluwer Academic, Dordrecht, 1999.

2. R. P. Agarwal, Huei-Lin Hong and Cheh-Chih Yeh, The Existence of Positive Solutions for the Sturm-Liouville Boundary Value Problems, Comput. Math. Appl., 35 (1998), 89-96.

3. D. Averna and G. Bonanno, A three critical points theorem and its applications to the ordinary Dirichlet problem, Topol. Methods Nonlinear Anal., 22 (2003), 93-104.

4. D. Averna and G. Bonanno, Three solutions for a quailinear two-point boundary value problem involving the one-dimensional $p$-Laplacian, Proc. Edinb. Math. Soc., 47 (2004), 257-270.

5. D. Averna and R. Salvati, Three solutions for mixed boundary value problem involving the one-dimensional p-Laplacian, J. Math. Anal. Appl., 298 (2004), 245-260.

6. R. I. Avery, Chyan Chuan Jen and J. Henderson, Twin solutions of boundary value problems for ordinary differential equations and finite difference equations, Comput. Math. Appl., 42 (2001), 695-704.

7. R. I. Avery and J. Henderson, Three Symmetric Positive Solutions for a Second-Order Boundary Value Problem, Appl. Math. Lett., 13 (2000), 1-7.

8. G. Bonanno, Existence of three solutions for a two point boundary value problem, Appl. Math. Lett., 13 (2000), 53-57.

9. G. Bonanno, A minimax inequality and its applications to ordinary differential equations, J. Math. Anal. Appl., 270 (2002), 210-229.

10. G. Bonanno and R. Livrea, Multiplicity theorems for the Dirichlet problem involving the p-Laplacian, Nonlinear Anal., 54 (2003), 1-7.

11. G. Bonanno, Multiple critical points theorems without the Palais-Smale condition, $J$. Math. Anal. Appl., 299 (2004), 600-614.

12. D. Guo and V. Lakshmikantham, Nonlinear Problems in Abstract cones, Academic Press, New York, (1988).

13. Yanping Guo and Weigao Ge, Three positive solutions for the one-dimensional pLaplacian, J. Math. Anal. Appl., 286 (2003), 491-508.

14. Xiaoming He and Weigao Ge, Existence of three solutions for a quasilinear two-point boundary value problem, Comput. Math. Appl., 45 (2003), 765-769. 
15. J. Henderson and H. B. Thompson, Existence of Multiple Solutions for Second Order Boundary Value Problems, J. Differential Equations, 166 (2000), 443-454.

16. L. H. Erbe and Haiyan Wang, On the existence of positive solutions of ordinary differential equation, Proc. Amer. Math. Soc., 120(3) (1994), 743-748.

17. R. W. Leggett and L. R. Williams, Multiple positive fixed poits of nonlinear operators on ordered Banach spaces, Indiana Univ. Math. J., 28 (1979), 673-688.

18. B. Ricceri, On a three critical points theorem, Arch. Math. (Basel), 75 (2000), 220-226.

19. B. Ricceri, A general variational principle and some of its applications, J. Comput. Appl. Math., 113 (2000), 401-410.

20. E. Zeidler, Nonlinear Functional Analysis and Its Applications, Vol. II/B, Springer, Berlin, 1990.

21. E. Zeidler, Nonlinear Functional Analysis and Its Applications, Vol. III, BerlinHeidelberg-New York, 1985.

22. R. Livrea, Existence of three solutions for a quasilinear two point boundary value problem, Arch. Math., 79 (2002), 288-298.

\author{
Yu Tian ${ }^{1,2}$ and Weigao $\mathrm{Ge}^{2}$ \\ ${ }^{1}$ School of Science, \\ Beijing University-f Posts and Telecommunications, \\ Beijing 100876, \\ P. R. China \\ E-mail: tianyu2992@163.com \\ ${ }^{2}$ Department of Applied Mathematics, \\ Beijing Institute of Technology, \\ Beijing 100081, \\ P. R. China
}

